\title{
Antibodies to MOG in adults with inflammatory demyelinating disease of the CNS
}

\section{OPEN}

Sung-Min Kim, MD,

PhD*

Mark R. Woodhall, PhD

Ji-Sun Kim, MD

Seong-Joon Kim, MD,

$\mathrm{PhD}$

Kyung Seok Park, MD,

$\mathrm{PhD}$

Angela Vincent, FRS

Kwang-Woo Lee, MD,

$\mathrm{PhD}$

Patrick Waters, PhD*

Correspondence to

Dr. Sung-Min Kim:

sueh916@gmail.com or

Dr. Waters:

paddy.waters@ndcn.ox.ac.uk

Supplemental data at Neurology.org/nn

\section{ABSTRACT}

Objective: To evaluate the clinical relevance of myelin oligodendrocyte glycoprotein antibody (MOG-Ab) in a cohort of adults with inflammatory demyelinating disease (IDD) of the CNS.

Methods: Live cell-based assays for MOG-Ab (lgG1 subset) and antibody to aquaporin-4 (AQP4Ab) were performed in a cohort of 270 adult patients with IDD and 72 controls. Patients were first grouped by positive antibody result as MOG-Ab or AQP4-Ab, and the remainder were grouped by published diagnostic criteria.

Results: Seventeen patients with IDD (6.3\%) had MOG-Abs and 49 patients (18.1\%) had AQP4Abs; none had both antibodies. The MOG-Ab patients predominantly manifested with isolated symptoms of optic neuritis (83\%). One-third of these patients experienced relapses, which involved only the optic nerve, and all relapsed within 1 year of disease onset. At onset, MRI in the MOG-Ab group uniquely demonstrated perineural enhancement, extending to the soft tissues around the optic nerves (33\%). Although about 30\% of MOG-Ab patients had brain MRI lesions, they had fewer periventricular lesions than the 26 patients with relapsing-remitting multiple sclerosis (MS); none of these lesions were ovoid or perpendicular to the ventricle. Moreover, MOG-Ab patients did not meet the diagnostic criteria for definite neuromyelitis optica (NMO) and had less spinal cord involvement than the AQP4-Ab group. Four patients (23.5\%) had poor visual outcomes (<0.2) or paraplegia.

Conclusions: MOG-Abs may be a disease-specific biomarker in adult patients with IDD who have a disease distinct from NMO or MS. The radiologic as well as clinical manifestations of MOG-Ab patients can be useful in their differential diagnosis. Neurol Neuroimmunol Neuroinflamm 2015;2:e163; doi: $10.1212 / \mathrm{NXI} .0000000000000163$

\section{GLOSSARY}

$\mathbf{A b}=$ antibody; $\mathbf{A D E M}=$ acute disseminated encephalomyelitis; AQP4 = aquaporin-4; $\mathbf{A T M}=$ acute transverse myelitis; CIS = clinically isolated syndrome; EDSS = Expanded Disability Status Scale; IDD = inflammatory demyelinating disease; MOG = myelin oligodendrocyte glycoprotein; $\mathbf{M S}=$ multiple sclerosis; $\mathbf{N M O}=$ neuromyelitis optica; NMOSD = NMO spectrum disorder; $\mathbf{O N}=$ optic neuritis; $\mathbf{V A}$ = visual acuity.

Inflammatory demyelinating diseases (IDDs) of the CNS are a group of heterogeneous autoimmune inflammatory diseases that include multiple sclerosis (MS), ${ }^{1}$ neuromyelitis optica $(\mathrm{NMO}),{ }^{2}$ acute disseminated encephalomyelitis (ADEM), ${ }^{3}$ optic neuritis (ON), ${ }^{4}$ and acute transverse myelitis (ATM). ${ }^{5}$ Autoantibodies to aquaporin-4 (AQP4-Abs), specific to NMO, were discovered a decade ago $^{6}$ and have greatly facilitated the identification of clinical manifestations of $\mathrm{NMO},{ }^{7}$ the differentiation of NMO spectrum disease (NMOSD) from MS, ${ }^{8}$ and the use of more-appropriate treatments. ${ }^{9}$

Several recent studies have shown the presence of myelin oligodendrocyte glycoprotein antibody (MOG-Ab) in the serum of adult patients with the NMOSD phenotype. ${ }^{10,11}$ However, the clinical relevance of MOG-Abs among adult patients with IDD is not yet clear. ${ }^{12}$ In this

\footnotetext{
*These authors contributed equally to the manuscript.

From the Department of Neurology (S.-M.K., J.-S.K., K.S.P., K.-W.L.) and Ophthalmology (S.-J.K.), Seoul National University College of Medicine, Seoul, Republic of Korea; Nuffield Department of Clinical Neurosciences (M.R.W., A.V., P.W.), John Radcliffe Hospital, Oxford, United Kingdom; and Department of Neurology (K.S.P.), Seoul National University Bundang Hospital, Gyeonggi, Republic of Korea.

Funding information and disclosures are provided at the end of the article. Go to Neurology.org/nn for full disclosure forms. The Article Processing Charge was paid by the authors.

This is an open access article distributed under the terms of the Creative Commons Attribution-NonCommercial-NoDerivatives License 4.0 (CC BY-NC-ND), which permits downloading and sharing the work provided it is properly cited. The work cannot be changed in any way or used commercially.
} 

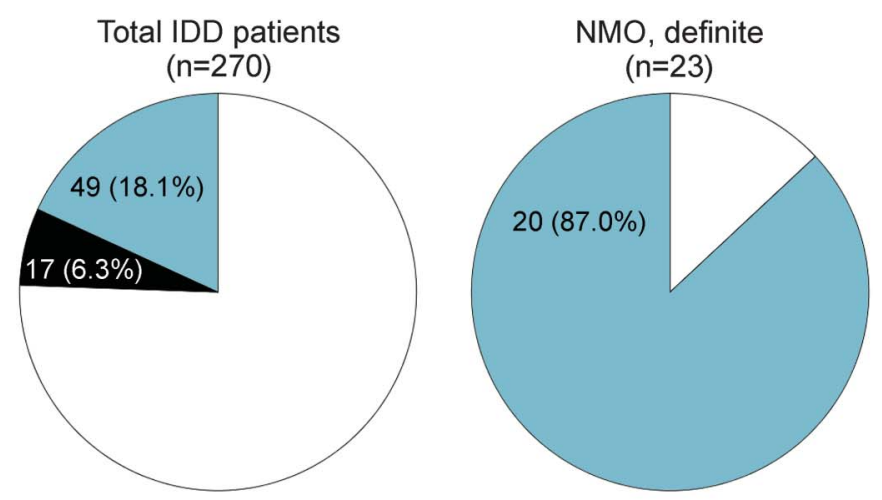

ON, recurrent or bilateral $(n=30)$
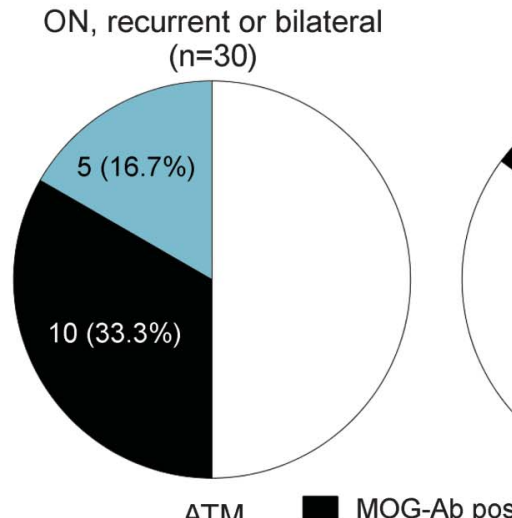

ON, single attack $(n=27)$
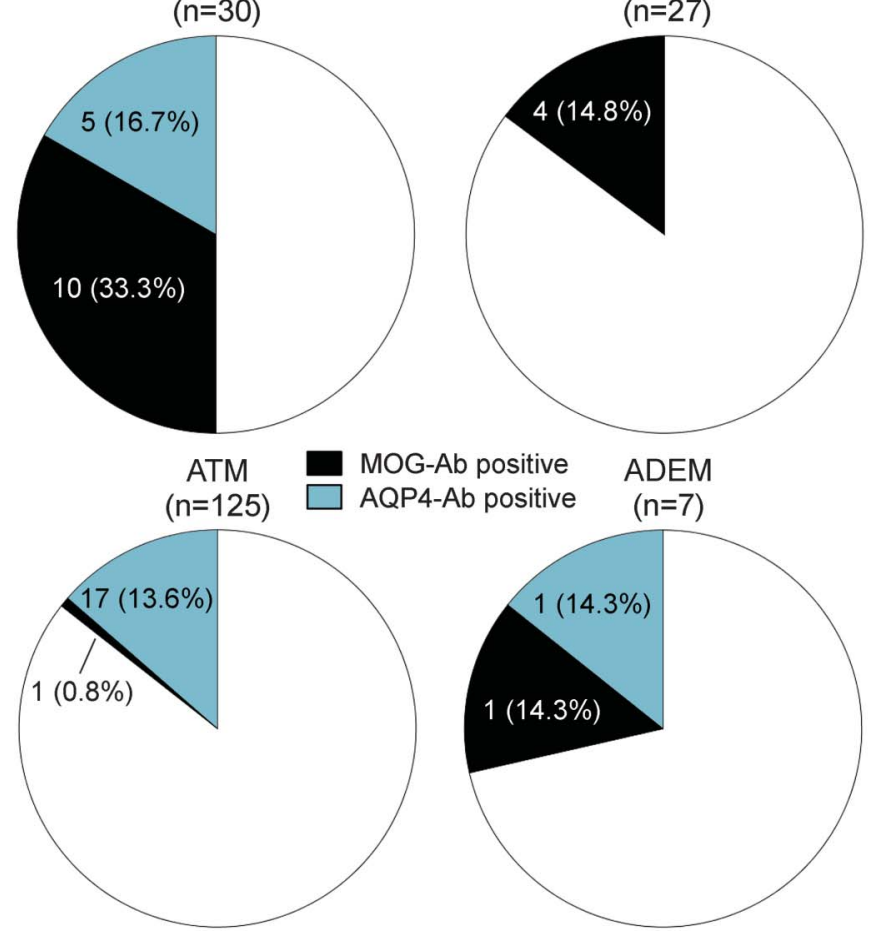

Ab positive
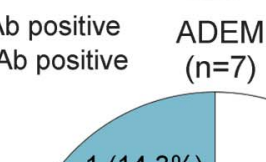

$(n=7)$

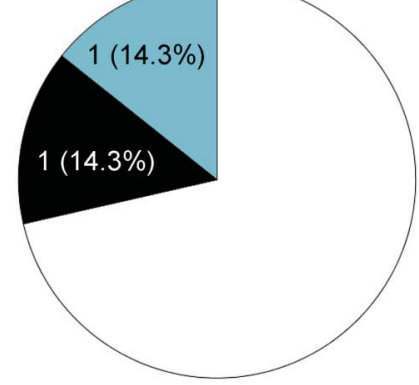

Overall, $6.3 \%$ of patients with inflammatory demyelinating disease were myelin oligodendrocyte glycoprotein antibody (MOG-Ab) positive (black) and $18.1 \%$ were aquaporin- 4 antibody (AQP4-Ab) positive (blue). The majority of MOG patients had optic neuritis (ON), whereas the majority of AQP4 patients had either neuromyelitis optica (NMO) or acute transverse myelitis (ATM). Just over $50 \%$ of patients with recurrent or bilateral ON were antibodypositive. ADEM = acute disseminated encephalomyelitis.

study, a large number of sera from adults with IDD and controls were tested for antibodies to MOG and AQP4 by cell-based assays. ${ }^{13}$ The clinical features of patients with MOG-Abs are described and compared to those of patients with AQP4-Abs or MS.

METHODS Patients and controls. The study population (IDD group) consisted of 270 consecutive patients with IDD who were suspected of having $\mathrm{MS},{ }^{1}$ definite $\mathrm{NMO}^{2}$ or clinical features of NMOSD, ${ }^{7} \mathrm{ADEM},{ }^{3}$ clinically isolated syndrome (CIS), ${ }^{14}$ ATM,, 5 or ON. ${ }^{15}$ This study also included 72 controls with noninflammatory diseases, including tumors $(\mathrm{n}=15)$, vascular disease ( $n=11)$, peripheral neuropathy $(n=7)$, encephalitis $(n=5)$, metabolic disease $(n=5)$, ocular disease $(n=5)$, degenerative spinal disease $(n=5)$, hereditary disease $(n=4)$, infection $(\mathrm{n}=3)$, psychogenic problems $(\mathrm{n}=3)$, motor neuron disease $(n=2)$, and others $(n=7)$.

All patients were older than 16 years $^{16}$ and visited Seoul National University Hospital MS/NMO Clinic (IDD group) or Neurology Clinic (control group) between November 2010 and October 2013. All patients were followed for more than 6 months.

Assay for AQP4-Ab and MOG-Ab. Samples were immediately centrifuged, stored at $-80^{\circ} \mathrm{C}$ according to the standard protocols,${ }^{16}$ and shipped on dry ice to the John Radcliffe Hospital, Oxford, UK. Only serial study numbers and initials of the patients were provided, and the diagnoses and epidemiologic data were unknown to the testing personnel (M.R.W., P.W.). The samples were analyzed for binding to $\mathrm{AQP} 4^{17}$ and $\mathrm{MOG}^{13}$ using cell-based assays. Antibodies to full-length MOG were detected with anti-human IgG1.

Classification of patients. Patients were grouped based on antibody test results as the MOG group, AQP4 group, double-positive group, or seronegative group (figure e-1 at Neurology.org/nn). In the seronegative group, those meeting the international panel criteria for relapsing-remitting $\mathrm{MS}^{1}$ were further classified as the MS group. Patients were also classified according to diagnostic criteria for definite $\mathrm{NMO},{ }^{2} \mathrm{ADEM},{ }^{3} \mathrm{ATM},{ }^{5}$ or $\mathrm{ON} .{ }^{15}$ Two neurologists (S.-M.K. and J.-S.K.) independently assessed the diagnoses of the patients according to their symptoms and published criteria. ${ }^{1-3,5,15}$ For any diagnostic disagreement, discussion and reassessment of the medical record were made to achieve consensus.

Comparison of the clinical, radiologic, and prognostic characteristics. Epidemiologic data, frequency of attacks, involved structures, patterns of MRI abnormalities, CSF analysis, disability and severity, response to treatment, and disease courses were assessed blinded to the antibody status. The frequency of attack was measured only in patients who were followed for more than a year. Maximal Kurtzke Extended Disability Status Scale (EDSS) score $^{18}$ at the nadir of disease and at last follow-up were assessed. A perineural enhancement pattern of the optic nerve was defined as an extensive enhancement pattern that was not limited to the optic nerve but extended to the soft tissues of the orbit. A relapse was defined as an episode of a new neurologic deficit that lasted at least 24 hours and occurred more than 1 month apart from a previous attack. An exacerbation was defined as an aggravation in the symptoms of a previous attack that lasted at least 24 hours, followed a period of nadir or recovery of a previous attack, was not attributable to fever or fatigue, and occurred within 1 month from the onset of a previous attack. A steroid-dependent relapse or exacerbation was defined as a relapse or exacerbation that occurred within 1 month from the cessation or dose reduction of oral steroid.

Statistical analysis. The Kruskal-Wallis test followed by pairwise comparison, or one-way analysis of variance followed by Tukey test were performed to compare the clinical characteristics between the MOG-Ab, AQP4-Ab, and MS groups. The significance level was set at $p<0.05$. Analyses were performed using SPSS software (version 22; SPSS, Chicago, IL).

Standard protocol approvals, registrations, and patient consents. The study was approved by the Seoul National University Hospital Institutional Review Board (approval number: H-1012-080-344). All patients provided written informed consent before participating. 
Table 1 Comparison of clinical, radiologic, and prognostic characteristics of MOG group with MS and AQP4 group

\begin{tabular}{|c|c|c|c|c|c|}
\hline & \multirow[b]{2}{*}{ MOG group } & \multirow[b]{2}{*}{ MS group } & \multirow[b]{2}{*}{ AQP4 group } & \multicolumn{2}{|l|}{$p$ Value } \\
\hline & & & & MOG vs MS & MOG vs AQP4 \\
\hline Sex, M:F & $7: 10$ & 12:14 & $7: 42$ & NS & NS \\
\hline Age at onset, $y$, mean $\pm S D(\min -\max )$ & $\begin{array}{l}42.69 \pm 19.3(18.12- \\
70.69)\end{array}$ & $\begin{array}{l}32.8 \pm 10.0(19.2- \\
58.6)\end{array}$ & $\begin{array}{l}43.41 \pm 13.96 \text { (16.43- } \\
75.3)\end{array}$ & NS & NS \\
\hline \multicolumn{6}{|l|}{ Attacks per year, mean \pm SD (min-max) } \\
\hline Total follow-up & $1.7 \pm 1.2(1-5)$ & $3.4 \pm 2.4(1-11)$ & $3.4 \pm 2.1(1-14)$ & 0.034 & 0.012 \\
\hline During first year of disease onset & $1.4+0.7(1-3)$ & $1.5+0.1(1-2)$ & $1.6+0.1(1-4)$ & NS & NS \\
\hline Frequency of attack, per year & $0.7 \pm 0.7(0.2-2.8)$ & $0.6 \pm 0.4(0.2-1.6)$ & $0.6 \pm 0.4(0.1-2.0)$ & NS & NS \\
\hline Spinal cord & $0.07 \pm 0.21(0-0.78)$ & $0.2 \pm 0.3(0-1.6)$ & $0.4 \pm 0.4(0-1.6)$ & NS & 0.006 \\
\hline Brain & $0.17 \pm 0.28(0-0.79)$ & $0.4 \pm 0.3(0-1.1)$ & $0.1 \pm 0.2(0-0.6)$ & 0.049 & NS \\
\hline
\end{tabular}

Involved structure, $\mathrm{n}(\%)$

Disease onset

Optic nerve

Spinal cord

Brain

Relapse $^{a}$

Optic nerve

Spinal cord

Brain

Either brain or spinal cord

Clinical symptomatic manifestations, $n(\%)$

Definite NMO
Clinical features of other NMOSD

Isolated optic neuritis

Isolated myelitis

Isolated brain syndrome

MRI findings, $n(\%)$

Presence of brain abnormality
Periventricular lesion
Ovoid lesions perpendicular to the ventricles
Large $(>3 \mathrm{~cm}$ ) brain lesion
Perineural enhancement of optic nerve
Maximal contiguous length of myelitis,
mean \pm SD (min-max)
Conus medullaris abnormality
CSF study, mean \pm SD (min-max)

White blood cells, per $\mathrm{mm}^{3}$

CSF/serum albumin ratio, $\times 10^{3}$

CSF/serum IgG ratio

CSF protein

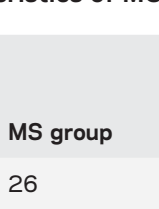

with MS and AQP4 group

p Value

MOG vs MS MOG vs AQP4

$0.17 \pm 0.28(0-0.79)$

$0.4 \pm 0.3(0-1.1)$

$0.1 \pm 0.2(0-0.6)$

0.049

5 (19.2)
$7(26.9)$
$14(53.9)$

7 (30.4)

11 (47.8)

11 (47.8)

20 (87.0)

$\begin{array}{ll}0 & 0 \\ 12(71) & 4(15) \\ 14(83) & 0 \\ 1(5.9) & 3(11) \\ 1(5.9) & 6(23)\end{array}$

5 (29)

2 (12)

0

2 (12)

6 (33)

$8.5 \pm 0.7(8-9)$

$1(5.9)$

$33.46 \pm 83.55(0-300)$

$5.3 \pm 4.0$ (1.6-15.5)

$4.5 \pm 4.6$ (1.1-17.2)

$47.2 \pm 46.9(21-191)$

0

0

26 (100)

25 (96)

$21(80.7)$

3 (11.5)

$18(36.7)$
$24(49.0)$
$12(24.5)$
$22(50)$
$37(84.1)$
$13(29.5)$
$39(88.6)$

0.000

NS

NS

NS

0.021

0.006

0.000

19 (39)

25 (52)

4 (8.2)

14 (28.6)

2 (4.1)

28 (58.3)

12 (25)

7 (14.6)

5 (10.4)

0

$2.2 \pm 1.4(1-5)$

$(1-5)$

NS

0.001

0.000

NS

NS

0.003

0.018

NS

NS

0.000

NS

0.000

0.002

NS

0.000

NS

NS

NS

$0.000 \quad N S$

$0.000 \quad$ NS

NS NS

$0.000 \quad 0.000$

$0.032 \quad N S$

NS NS

(n)

$\begin{array}{llll}4.88 \pm 5.63(0-18) & 8.27 \pm 11.08(0-47) & \text { NS } & \text { NS } \\ 4.7 \pm 2.8(0-9.1) & 7.2 \pm 3.2(3.2-20.8) & \text { NS } & \text { NS } \\ 3.2 \pm 1.5(1.3-6.2) & 3.7 \pm 1.7(1.8-9.5) & \text { NS } & \text { NS } \\ 32.8 \pm 14.7(16-59) & 47.1 \pm 23.7(18-122) & \text { NS } & \text { NS }\end{array}$

Continued 


\begin{tabular}{|c|c|c|c|c|c|}
\hline \multicolumn{6}{|l|}{ Table 1 Continued } \\
\hline & \multirow[b]{2}{*}{ MOG group } & \multirow[b]{2}{*}{ MS group } & \multirow[b]{2}{*}{ AQP4 group } & \multicolumn{2}{|l|}{$p$ Value } \\
\hline & & & & MOG vs MS & MOG vs AQP4 \\
\hline \multicolumn{6}{|l|}{ Disability and severity } \\
\hline EDSS at first attack, mean \pm SD (min-max) & $3.96 \pm 1.96(2-8)$ & $2.55 \pm 1.74(1-6.4)$ & $4.67 \pm 2.82(1-9.5)$ & NS & NS \\
\hline Patients with EDSS $\geq 6$ at first attack, $n(\%)$ & 2 (12.5) & $3(13.0)$ & $13(28.9)$ & NS & NS \\
\hline Patients with EDSS $\geq 6$ at last follow-up, $n$ (\%) & $1(5.9)$ & 0 & 5 (10.2) & NS & NS \\
\hline Death, n (\%) & 0 & 0 & $2(4.1)$ & NS & NS \\
\hline \multicolumn{6}{|l|}{ Treatment, $\mathrm{n}$ (\%) } \\
\hline Plasmapheresis for acute attack & 4 (23.5) & $2(7.7)$ & $13(26.5)$ & NS & NS \\
\hline Azathioprine & $3(17.7)$ & $3(11.5)$ & $27(55.1)$ & NS & 0.017 \\
\hline Oral prednisone alone & $2(11.8)$ & 1 (3.9) & 6 (12.2) & NS & NS \\
\hline Rituximab & $1(5.9)$ & 0 & $16(32.7)$ & NS & 0.044 \\
\hline Any disease-modifying treatment & 4 (23.5) & 25 (96.2) & $43(87.8)$ & 0.000 & 0.000 \\
\hline \multicolumn{6}{|l|}{ Relapse or exacerbation } \\
\hline $\begin{array}{l}\text { Presence of steroid-dependent relapse or } \\
\text { exacerbation, } n(\%)\end{array}$ & 4 (23.5) & 0 & $11(22.5)$ & NS & NS \\
\hline Presence of relapse during follow-up, $n(\%)$ & $5(29.4)$ & $23(88)$ & $44(89.8)$ & 0.000 & 0.000 \\
\hline
\end{tabular}

Abbreviations: AQP4 = aquaporin-4; EDSS = Extended Disability Status Scale; MOG = myelin oligodendrocyte glycoprotein; MS = multiple sclerosis; $\mathrm{NMO}=$ neuromyelitis optica; NMOSD = NMO spectrum disorder; NS = not significant.

${ }^{\text {a}}$ Estimated among patients with relapsing courses.

RESULTS Classification of patients and frequencies of MOG-Ab and AQP4-Ab. Overall, serum antibodies in $17 / 270(6.3 \%)$ of the IDD patients bound to MOG (MOG group) and antibodies in 49/270 (18.1\%) bound to AQP4 (AQP4 group), with no double-positives. None of the controls were positive for either MOG-Ab or AQP4-Ab. Twenty-six of the 204 seronegative IDD patients fulfilled the international panel criteria for relapsing-remitting MS (MS group). Others were classified as having seronegative $\mathrm{NMO}(\mathrm{n}=3)$, primary progressive MS ( $\mathrm{n}=3)$, ADEM $(\mathrm{n}=5), \operatorname{ATM}(\mathrm{n}=107)$, ON ( $\mathrm{n}=38)$, or CIS $(\mathrm{n}=22)$, which was similar to a previous study on Korean IDD. ${ }^{17}$

MOG-Abs were most frequent in patients with recurrent or bilateral $\mathrm{ON}(10 / 30 ; 33.3 \%)$ or those with a single ON attack $(4 / 27 ; 14.8 \%)$, whereas only $1 / 125$ patients with ATM (0.8\%), 1/7 with ADEM (14.3\%), and none with definite NMO ( $\mathrm{n}=23$ ) had MOG-Abs. AQP4-Ab positivity was found in patients with definite NMO (20/23; $87.0 \%)$ but also in patients with recurrent or bilateral ON $(5 / 30 ; 16.7 \%)$, ADEM $(1 / 7 ; 14.3 \%)$, or $\operatorname{ATM}(17 / 125 ; 13.6 \%)$. The assay results are summarized in figure e-1, and the proportion of each disease group with MOG- and AQP4-Abs is shown in figure 1 .

Characteristics of IDD patients with MOG-Ab. The most common symptomatic clinical manifestation in the MOG group was isolated ON (83.3\%; table 1).
Although most of these cases with isolated $\mathrm{ON}$ were recurrent or bilaterally simultaneous/consecutive, 4 of them manifested as a single attack of unilateral ON. The visual acuity (VA) was severely affected at nadir, with VA less than or equal to 0.2 in $75 \%(9 / 12)$ of patients. Perineural enhancement that extended around the soft tissue of the optic nerve, visualized by MRI, was found in 33\% of patients in the MOG group (table 1 and figure 2). This pattern of orbital inflammation was more prominent in their first $\mathrm{ON}$ attacks than in subsequent $\mathrm{ON}$ attacks (data not shown). Brain MRI abnormalities were found in only 5 (29\%) of these patients, and they were asymptomatic in 3 cases (figures 3 and 4). They tended to be large $(>3 \mathrm{~cm})$ and involve white matter and the internal capsule (figure 3, A and B); in some cases, they mimicked the lesions of posterior reversible encephalopathy (figure 3, C and D). There were also symmetric brainstem lesions involving pontine tegmentum (figure 3, E-G). There were no ovoid lesions or lesions perpendicular to the ventricles as typically found in MS.

Only 2 MOG-Ab patients (11\%) had spinal cord involvement, but the lesions were very long (8-9 continuous vertebral segments).

MOG-Ab patients with poor outcomes. Although MOG-Ab patients have previously been shown to have a generally good recovery, ${ }^{10} 4$ of our 17 patients had severe sequelae, 3 from their first attack. Patient 6 had severe bilateral ON, causing 


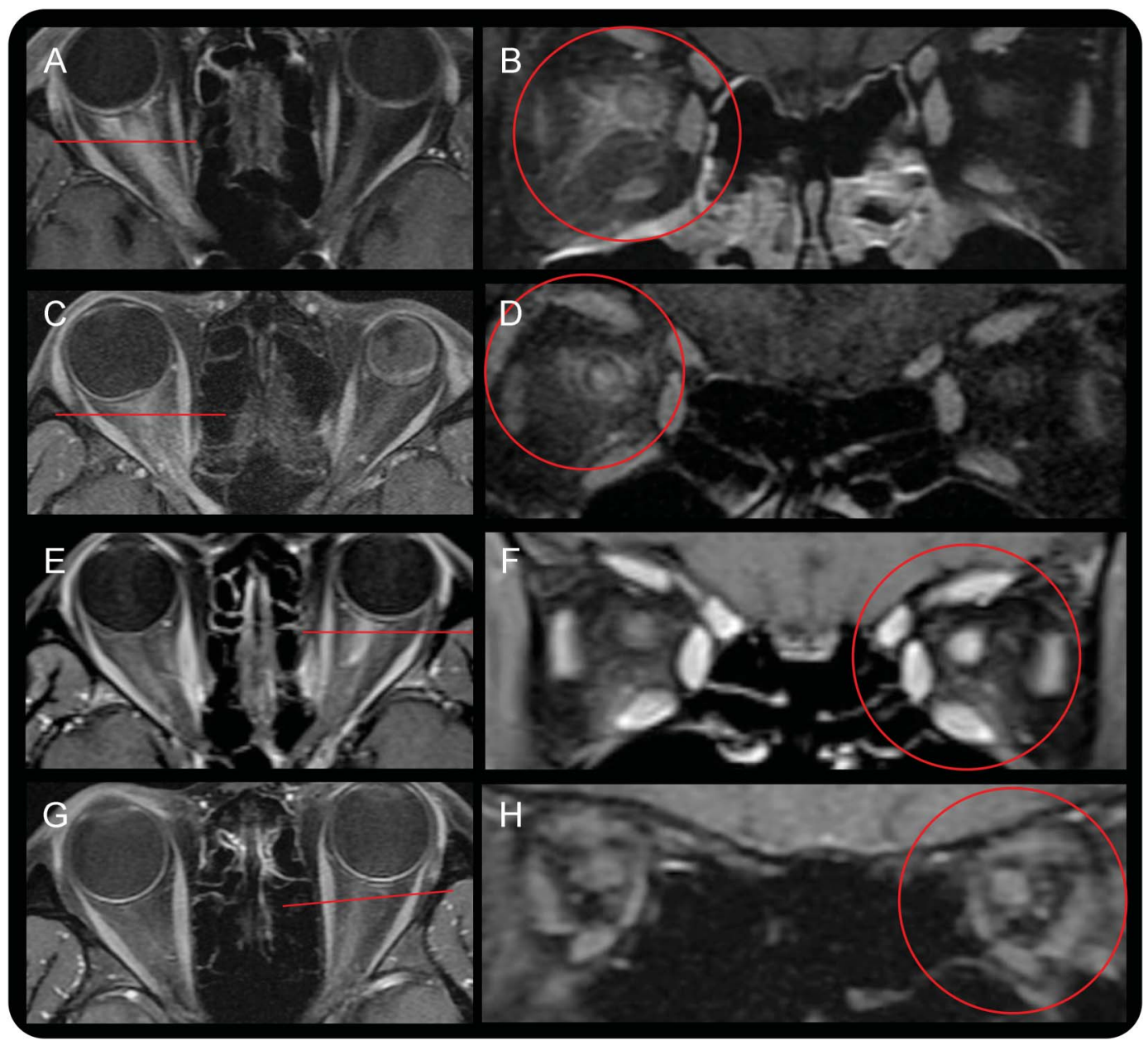

One-third of myelin oligodendrocyte glycoprotein antibody-positive patients revealed extensive enhancement patterns that were not confined to the optic nerve but extended to the soft tissues around the optic nerves (perineural enhancement) $(A-D)$, which were not observed in the neuromyelitis optica group ( $E$ and $F$ ) or the multiple sclerosis group ( $G$ and $H$ ). All MRIs were T1-weighted with gadolinium enhancement. Red lines in $A, C, E$, and $G$ highlight the level where the transverse images in $\mathrm{B}, \mathrm{D}, \mathrm{F}$, and $\mathrm{H}$ are taken.

VA of 0.02 in the better eye. Although her VA improved after steroid treatment, it remains poor (0.15) after recovery. Patient 11 was initially misdiagnosed as having ischemic optic neuropathy and hence was not treated with steroids or plasmapheresis; she was referred to us after several months but was left with a poor VA of less than 0.2 bilaterally. Patient 17 became completely paraplegic after the first attack of longitudinally extensive myelitis, which involved the conus medullaris. Aggressive acute-phase treatment, including highdose methylprednisolone, plasmapheresis, and cyclophosphamide, was given, but he remains wheelchair-dependent nearly 4 years later. Patient 1 experienced 5 attacks of ON within 2 years of onset, which led to a confirmed VA of only hand motion perception in the right eye despite treatment with azathioprine and oral prednisolone. $\mathrm{He}$ was switched to rituximab to prevent further relapses and further loss of vision in the left eye and has been relapse free for 7 months (table e-1 and figure 4).

Comparison of the clinical, radiologic, and prognostic characteristics between groups. There was no difference in patient age at onset, but the well-recognized female predominance in AQP4 patients is reproduced here (table 1).

ON was more common in MOG-Ab patients than in the other 2 groups, and perineural enhancement of the optic nerve was found only in MOG$\mathrm{Ab}$ patients. They also had fewer relapses and were more likely to have a monophasic disease course (no relapse within 2 years). All 11 relapses in the 5 relapsing patients with MOG-Abs involved only the optic nerve, and 4 of 5 made an excellent recovery. In contrast, there was significantly more brain or spinal cord involvement in relapses in the other 2 groups $(87.0 \%$ in the MS group, $86.7 \%$ in the AQP4 group). 


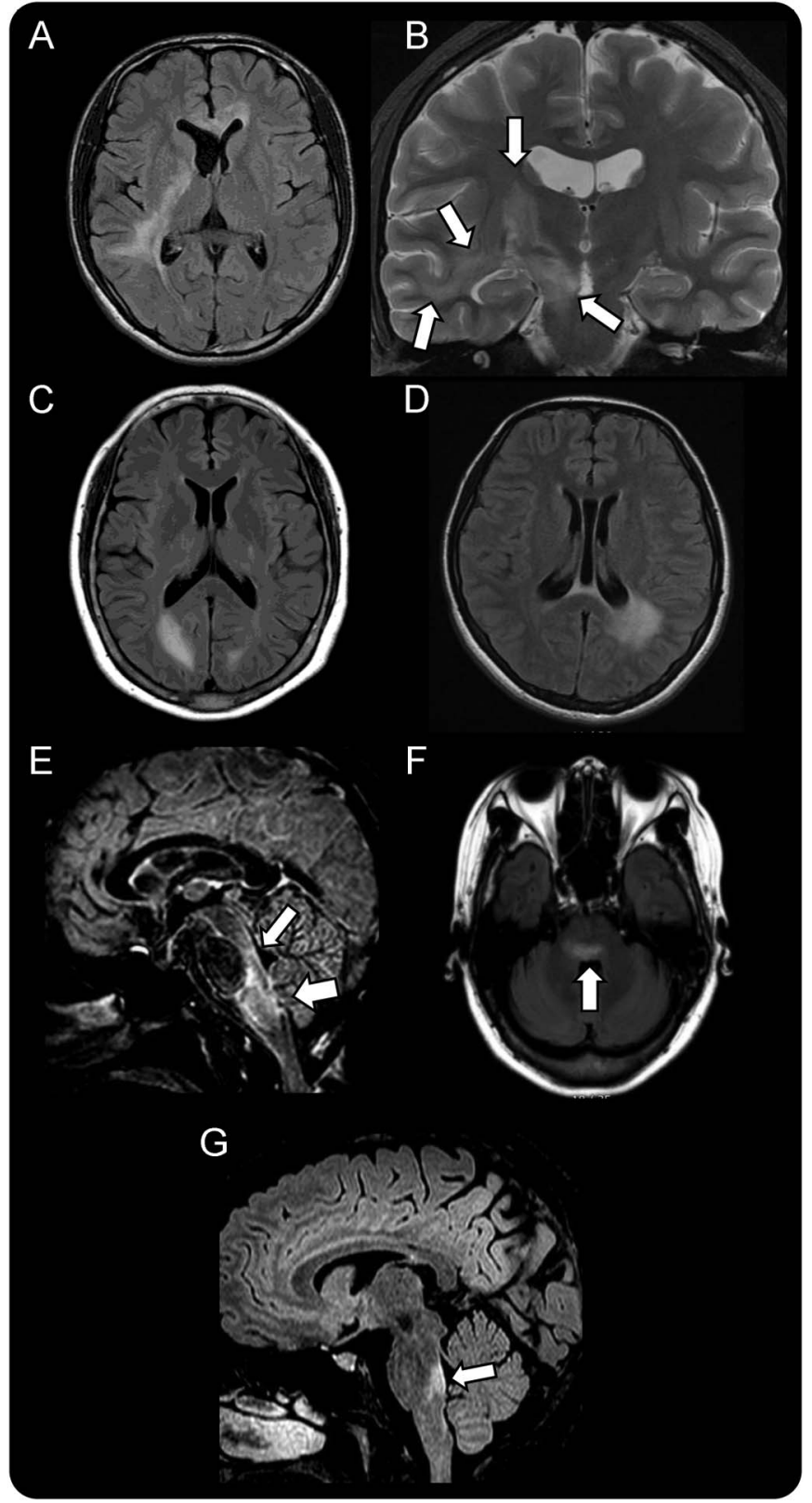

Extensive brain lesions with large diameter (A and B), posterior reversible encephalopathylike lesions $(C$ and $D)$, and symmetric brainstem lesions involving pontine tegmentum (E-G) were found in the myelin oligodendrocyte glycoprotein antibody (MOG-Ab) group. A, C, D, F, and $\mathrm{G}=$ fluid-attenuated inversion recovery; $\mathrm{B}=\mathrm{T} 2$-weighted image; $\mathrm{E}=$ double inversion recovery.
On brain MRI, the MOG group had fewer brain abnormalities, fewer periventricular lesions, and fewer ovoid lesions perpendicular to the ventricle than the MS group. Surprisingly, none of the MOG group met the 2006 Wingerchuk criteria for NMO, and the MOG group had fewer patients with spinal cord involvement than the AQP4 group.

However, there was no difference in the number of relapses during the onset year in the 3 groups, no difference in onset EDSS between MOG-Ab patients and those with MS or AQP4-Ab (table 1), and no difference in the number of patients who had an EDSS score $\geq 6$ at onset or at the last follow-up. The severe disability in the MOG group resulted from the initial attack in 3 of 4 cases (table e-1). The MOG group had a relatively short mean follow-up time of 3 years, but fewer patients in this group received any treatment $(5 / 17 ; 29.4 \%)$ compared to the AQP4 (43/49; $96.2 \%)$ and MS groups (25/26; 96.2\%).

DISCUSSION The potential significance of MOGAbs, measured against full-length MOG expressed in human cells, is only just beginning to be appreciated. In adult patients with IDD, these antibodies have been reported in a proportion of AQP4-Ab-negative patients with clinical features of NMOSDs. ${ }^{10,11}$ However, there are still concerns about the ability of MOG serostatus to distinguish between MS and non-MS conditions and doublepositive patients.

In this unselected study of 270 adult patients with IDD, just over $6 \%$ had IgG1 MOG-Abs, another $18.1 \%$ had AQP4-Abs, and none had both antibodies. Patients with MOG-Abs had a disease distinct from MS and NMO that is highlighted by differences in clinical manifestations, radiologic findings, treatment responses, and prognosis. Although the MOG-Abs seem to define a disease with a generally good prognosis, some patients $(4 / 17 ; 23.5 \%)$ were left with severe functional impairments, including complete paraplegia.

The IDD patients with MOG-Abs presented more often with $\mathrm{ON}$, more frequently manifested with symptoms of isolated ON (83\%), and had a more monophasic course $(71 \%)$ than those in the MS and AQP4-Ab groups. Particularly striking was the perineural enhancement of the optic nerve that extended to the soft tissues around the optic nerve, which was not observed in the MS or AQP4 groups and which was most evident in the first attack. Steroiddependent relapses or exacerbations were found in $20 \%$ of patients in the MOG group but not in the MS group. All patients with MS had brain lesions (38\% had ovoid lesions perpendicular to the ventricles) compared to less than half of the MOG patients, none of whom had MS-typical brain lesions ${ }^{19}$ but instead had large-diameter lesions or symmetric brainstem lesions involving the pontine tegmentum. Although spinal cord lesions were less common in the MOG group, the lesions in 2 patients were very extensive.

There were also differences between MOG-Ab and AQP4-Ab patients. MOG-Ab patients did not meet the revised criteria for definite $\mathrm{NMO} .^{10,11,2}$ In agreement with previous studies, we highlight the strong predilection of MOG-Abs for the optic nerve and less so for the spinal cord, unlike AQP4-Abs, which show opposite preferences (table 1). 


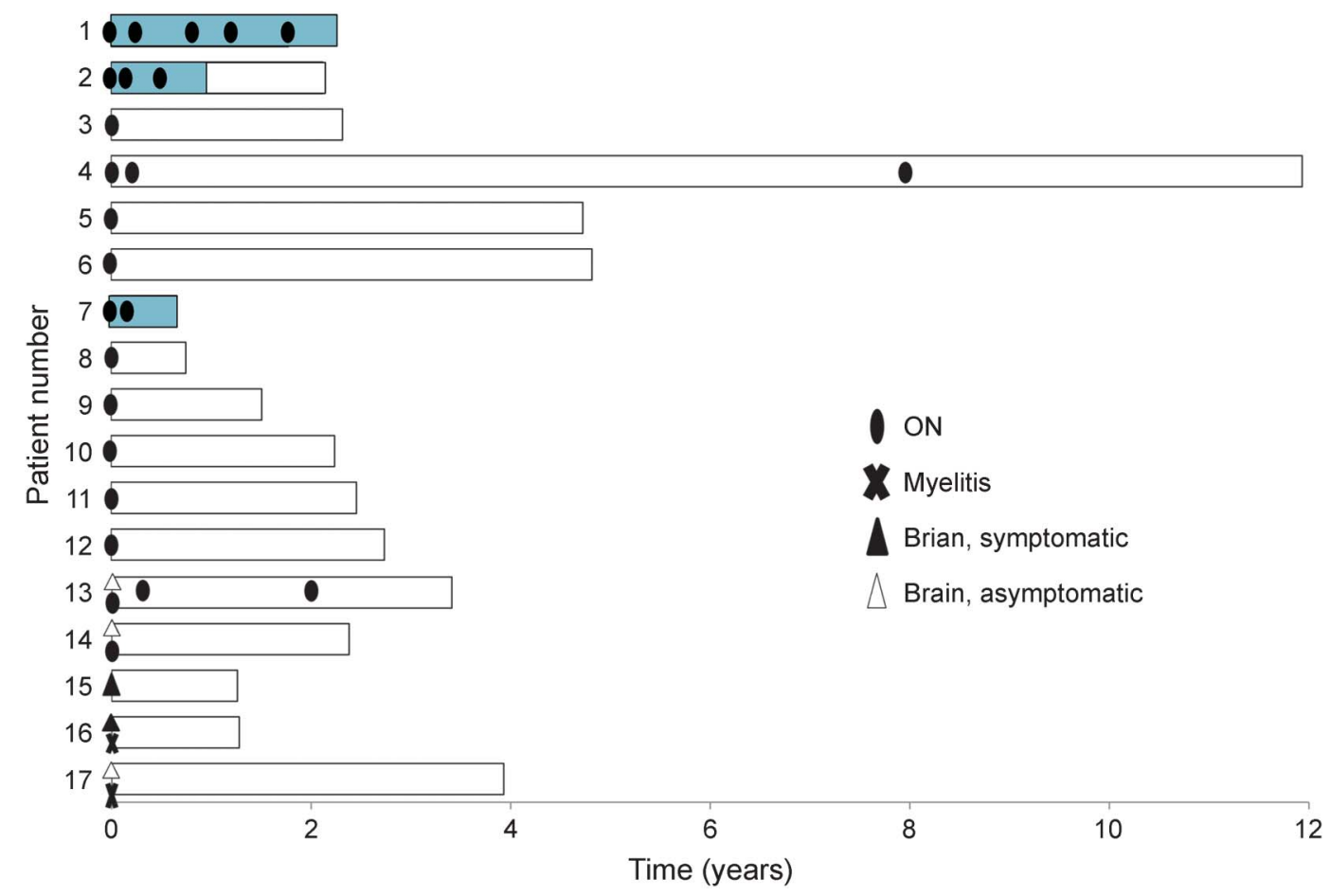

Five myelin oligodendrocyte glycoprotein antibody (MOG-Ab)-positive patients experienced a relapse, all of which manifested as optic neuritis (ON). All patients with a relapsing disease course experienced a relapse within the first year of disease onset. Blue bar represents treatment period for relapse prevention.

The majority of the patients with MOG-Abs had a monophasic course, as already suggested by previous studies. ${ }^{10,11}$ However, 5 (29\%) of our patients with MOG-Abs experienced relapses. All of these relapses involved only the optic nerves, and the first relapse always occurred within 1 year from disease onset. These repeated patterns of $\mathrm{ON}$ attacks in patients with MOG-Abs were partly consistent with a recent study on pediatric patients with MOG-Abs. ${ }^{20}$

Recently, 2 important studies have described the clinical characteristics of adult patients with MOGAbs. ${ }^{10,11}$ However, both of these studies aimed to characterize the features of NMOSD patients with MOG-Abs compared to those with AQP4-Abs in a cohort limited to patients with clinical features of NMOSDs. ${ }^{10,11}$ In this study we evaluated the clinical relevance of MOG-Abs in a large number of consecutive adults with IDD and identified some important radiologic features of MOG-Ab patients, including perineural enhancement patterns around the optic nerve and patterns of brain lesions. These findings suggest that adult IDD with MOG-Abs is a separate entity from NMO and MS.

The clinical relevance of MOG-Abs has already been studied in pediatric cohorts, which have shown that this antibody has a sensitivity of $40 \%$ for pediatric patients with ADEM. ${ }^{21,22}$ However, in our study, only 1 of 8 patients (patient 16 in the MOG group) with clinical features of ADEM had MOG-Abs ${ }^{3}$ (figure 1 and table e-1), suggesting that some MOG-Abpositive patients may present as clinical mimics of ADEM. As the optimal diagnostic criteria for ADEM is still debated, detection of MOG-Abs may be an important aspect to consider. ${ }^{23}$

Although MOG-Ab patients were previously known to have a generally good recovery, ${ }^{10} 4$ of our patients (patients 1, 6, 11, and 17) had severe functional impairment, either VA of less than 0.2 or paraplegia. Patient 1 , who experienced $5 \mathrm{ON}$ attacks in 2 years and became nearly monocularly blind, had to be treated with rituximab to prevent complete blindness in both eyes. He has remained relapse free for 7 months after treatment with rituximab. Patient 17 with ATM remains completely paraplegic despite treatment with plasmapheresis and cyclophosphamide. Because the disability at attack nadir and neurologic sequelae were quite variable among patients with MOG-Abs, further studies are needed to identify prognostic factors for these patients. Of interest, the most severe disability (EDSS score of 8 ) in our MOG group was observed in patient 17 , who had ATM involving the conus, which was highlighted in a previous study. ${ }^{10}$

This study has some limitations. Although the cohort screened was large, the number of MOG-Ab patients was relatively small. The average follow-up 
period of 3 years for the MOG group is short. The percentage of relapse-free MOG-Ab-positive patients is similar to previously published work, ${ }^{10}$ but only longer follow-up will tell whether the relapse-free patients $(72 \%)$ remain so.

Nevertheless, it now seems clear that MOG-Abs are a biomarker for adult patients with IDD that have distinct serologic, clinical, and radiologic features from patients with MS or AQP4-Ab patients, suggesting that MOG-Abs might mediate a distinct disease.

\section{AUTHOR CONTRIBUTIONS}

Sung-Min Kim drafted the manuscript, designed the study, performed statistical analysis, and analyzed the data. Mark R. Woodhall, Ji-Sun Kim, and Seong-Joon Kim contributed to the acquisition of data. Kyung Seok Park contributed to patient and data recruiting. Angela Vincent revised the manuscript and was involved in study design. Kwang-Woo Lee contributed to patient and data recruiting. Patrick Waters drafted the manuscript, designed the study, confirmed the statistical analysis, and analyzed the data.

\section{STUDY FUNDING}

P. Waters, A. Vincent, and M. R. Woodhall are supported by NHS National Specialised Commissioning Group for Neuromyelitis Optica, United Kingdom, and by the NIHR Oxford Biomedical Research Centre. This work is supported by Grant No. A121118 from the Korea Health Industry Development Institute Research Fund and 03-20151284 SNUH Research Fund.

\section{DISCLOSURE}

S.-M. Kim received research support from the Korea Health Industry Development Institute Research Fund and SNUH Research fund. M. R. Woodhall, J.-S. Kim, S.-J. Kim, and K.S. Park report no disclosures. A. Vincent is on the editorial board for Neurology; was an Associate Editor for Brain; holds patents with Oxford University for LGI1/CASPR2 antibodies, licensed to Euroimmun AG, and for GABAAR antibodies, in negotiation with Euroimmun AG; receives royalties from Athena Diagnositcs, Euroimmun AG, Blackwell Publishing, and Mac Keith Press; consulted for Athena Diagnostics; and received research support from NIHR. K.-W. Lee reports no disclosures. P. Waters received speaker honoraria from Biogen Idec Japan and Euroimmun AG, is a review editor for Frontiers in Molecular Innate Immunity, and holds a patent for assays for the detection of antibodies to lgi1, Caspr2, Tag-1, and GABAAR. Go to Neurology.org/nn for full disclosure forms.

Received June 22, 2015. Accepted in final form August 21, 2015.

\section{REFERENCES}

1. Polman $\mathrm{CH}$, Reingold SC, Banwell B, et al. Diagnostic criteria for multiple sclerosis: 2010 revisions to the McDonald criteria. Ann Neurol 2011;69:292-302.

2. Wingerchuk DM, Lennon VA, Pittock SJ, Lucchinetti CF, Weinshenker BG. Revised diagnostic criteria for neuromyelitis optica. Neurology 2006;66:1485-1489.

3. de Seze J, Debouverie M, Zephir H, et al. Acute fulminant demyelinating disease: a descriptive study of 60 patients. Arch Neurol 2007;64:1426-1432.

4. Shams P, Plant G. Optic neuritis: a review. Int MS J 2009; 16:82-89.

5. Transverse Myelitis Consortium Working Group. Proposed diagnostic criteria and nosology of acute transverse myelitis. Neurology 2002;59:499-505.

6. Lennon VA, Wingerchuk DM, Kryzer TJ, et al. A serum autoantibody marker of neuromyelitis optica: distinction from multiple sclerosis. Lancet 2004;364:2106-2112.
7. Wingerchuk DM, Lennon VA, Lucchinetti CF, Pittock SJ, Weinshenker BG. The spectrum of neuromyelitis optica. Lancet Neurol 2007;6:805-815.

8. Kim SM, Waters P, Woodhall M, et al. Utility of aquaporin-4 antibody assay in patients with neuromyelitis optica spectrum disorders. Mult Scler 2013;19: 1060-1067.

9. Papadopoulos MC, Bennett JL, Verkman AS. Treatment of neuromyelitis optica: state-of-the-art and emerging therapies. Nat Rev Neurol 2014;10:493-506.

10. Sato DK, Callegaro D, Lana-Peixoto MA, et al. Distinction between MOG antibody-positive and AQP4 antibody-positive NMO spectrum disorders. Neurology 2014;82:474-481.

11. Kitley J, Waters P, Woodhall M, et al. Neuromyelitis optica spectrum disorders with aquaporin-4 and myelinoligodendrocyte glycoprotein antibodies: a comparative study. JAMA Neurol 2014;71:276-283.

12. Reindl M, Di Pauli F, Rostasy K, Berger T. The spectrum of MOG autoantibody-associated demyelinating diseases. Nat Rev Neurol 2013;9:455-461.

13. Waters P, Woodhall M, O'Connor KC, et al. MOG cellbased assay detects non-MS patients with inflammatory neurologic disease. Neurol Neuroimmunol Neuroinflamm 2015;2:e89. doi: 10.1212/NXI.0000000000000089.

14. Miller D, Barkhof F, Montalban X, Thompson A, Filippi M. Clinically isolated syndromes suggestive of multiple sclerosis, part I: natural history, pathogenesis, diagnosis, and prognosis. Lancet Neurol 2005;4:281-288.

15. Beck RW, Cleary PA, Anderson MM Jr, et al. A randomized, controlled trial of corticosteroids in the treatment of acute optic neuritis. The Optic Neuritis Study Group. N Engl J Med 1992;326:581-588.

16. Teunissen CE, Petzold A, Bennett JL, et al. A consensus protocol for the standardization of cerebrospinal fluid collection and biobanking. Neurology 2009;73:19141922.

17. Kim SM, Waters P, Woodhall M, et al. Characterization of the spectrum of Korean inflammatory demyelinating diseases according to the diagnostic criteria and AQP4Ab status. BMC Neurol 2014;14:93. doi: 10.1186/ 1471-2377-14-93.

18. Kurtzke JF. Rating neurologic impairment in multiple sclerosis: an expanded disability status scale (EDSS). Neurology 1983;33:1444-1452.

19. Horowitz AL, Kaplan RD, Grewe G, White RT, Salberg LM. The ovoid lesion: a new MR observation in patients with multiple sclerosis. AJNR Am J Neuroradiol 1989;10:303-305.

20. Ketelslegers IA, Van Pelt DE, Bryde S, et al. Anti-MOG antibodies plead against MS diagnosis in an Acquired Demyelinating Syndromes cohort. Mult Scler Epub 2015 Feb 6.

21. Brilot F, Dale RC, Selter RC, et al. Antibodies to native myelin oligodendrocyte glycoprotein in children with inflammatory demyelinating central nervous system disease. Ann Neurol 2009;66:833-842.

22. Lalive PH, Hausler MG, Maurey $\mathrm{H}$, et al. Highly reactive anti-myelin oligodendrocyte glycoprotein antibodies differentiate demyelinating diseases from viral encephalitis in children. Mult Scler 2011;17:297-302.

23. Young NP, Weinshenker BG, Lucchinetti CF. Acute disseminated encephalomyelitis: current understanding and controversies. Semin Neurol 2008;28:84-94. 


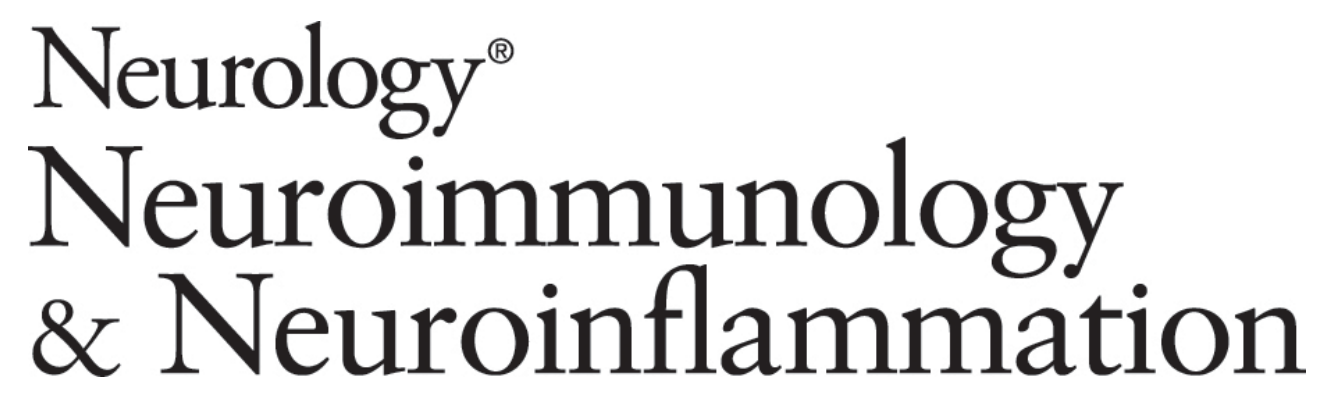

Antibodies to MOG in adults with inflammatory demyelinating disease of the CNS

Sung-Min Kim, Mark R. Woodhall, Ji-Sun Kim, et al.

Neurol Neuroimmunol Neuroinflamm 2015;2;

DOI 10.1212/NXI.0000000000000163

This information is current as of October 15, 2015

Neurol Neuroimmunol Neuroinflamm is an official journal of the American Academy of Neurology.

Published since April 2014, it is an open-access, online-only, continuous publication journal. Copyright $\odot$ 2015 American Academy of Neurology. All rights reserved. Online ISSN: 2332-7812.

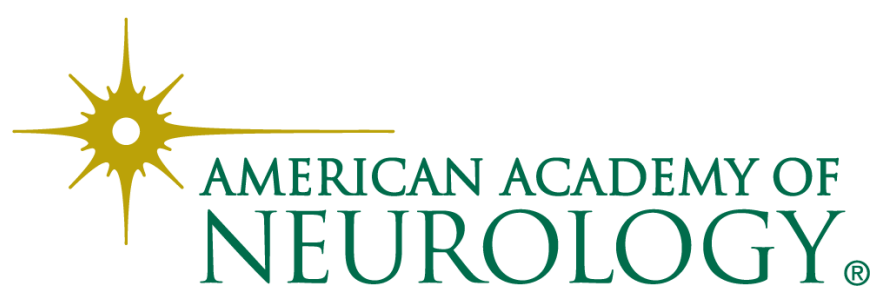




\section{Updated Information \& Services}

\section{Supplementary Material}

\section{References}

Citations

Subspecialty Collections

Permissions \& Licensing

Reprints including high resolution figures, can be found at: http://nn.neurology.org/content/2/6/e163.full.html

Supplementary material can be found at: http://nn.neurology.org/content/suppl/2015/10/15/2.6.e163.DC1

This article cites 22 articles, 0 of which you can access for free at: http://nn.neurology.org/content/2/6/e163.full.html\#\#ref-list-1

This article has been cited by 15 HighWire-hosted articles: http://nn.neurology.org/content/2/6/e163.full.html\#\#otherarticles

This article, along with others on similar topics, appears in the following collection(s):

Devic's syndrome

http://nn.neurology.org//cgi/collection/devics_syndrome

Multiple sclerosis

http://nn.neurology.org//cgi/collection/multiple_sclerosis Optic neuritis; see Neuro-ophthalmology/Optic Nerve http://nn.neurology.org//cgi/collection/optic_neuritis

Information about reproducing this article in parts (figures,tables) or in its entirety can be found online at:

http://nn.neurology.org/misc/about.xhtml\#permissions

Information about ordering reprints can be found online: http://nn.neurology.org/misc/addir.xhtml\#reprintsus

Neurol Neuroimmunol Neuroinflamm is an official journal of the American Academy of Neurology.

Published since April 2014, it is an open-access, online-only, continuous publication journal. Copyright $\odot$ 2015 American Academy of Neurology. All rights reserved. Online ISSN: 2332-7812.

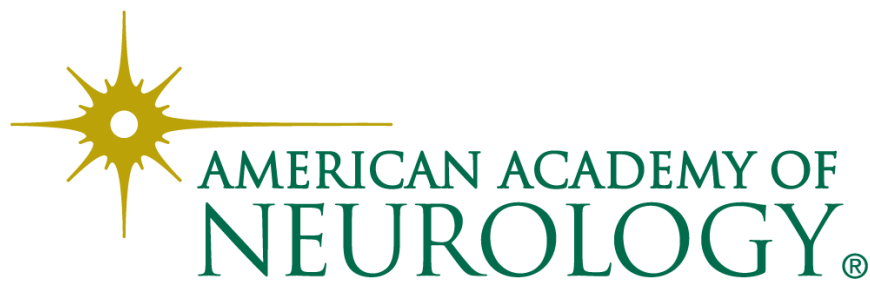

